\title{
The depth of the shower maximum of air showers measured with AERA
}

\author{
Bjarni Pont ${ }^{a, *}$ on behalf of the Pierre Auger ${ }^{b}$ Collaboration \\ (a complete list of authors can be found at the end of the proceedings) \\ ${ }^{a}$ Department of Astrophysics/IMAPP, Radboud University, P.O. Box 9010, NL-6500 GL Nijmegen, The \\ Netherlands \\ ${ }^{b}$ Observatorio Pierre Auger, Av. San Martín Norte 304, 5613 Malargüe, Argentina \\ E-mail: spokespersons@auger.org
}

The Auger Engineering Radio Array (AERA) is currently the largest array of radio antennas for the detection of cosmic rays, spanning an area of $17 \mathrm{~km}^{2}$ with 153 radio antennas, measuring in the energy range from $10^{17.0}$ to $10^{19.0} \mathrm{eV}$. It detects the radio emission of extensive air showers produced by cosmic rays in the $30-80 \mathrm{MHz}$ band. The cosmic-ray mass composition is a crucial piece of information in determining the sources of cosmic rays and their acceleration mechanisms. The depth of the shower maximum, $X_{\max }$, a probe for mass composition can be determined with a likelihood analysis that compares the measured radio-emission footprint on the ground to an ensemble of footprints from CORSIKA/CoREAS Monte-Carlo air shower simulations. These simulations are also used to determine the resolution of the method and to validate the reconstruction by identifying and correcting for systematic uncertainties. We will present the method for the reconstruction of the depth of the shower maximum, achieving a resolution of up to $15 \mathrm{~g} / \mathrm{cm}^{2}$, show compatibility with the independent fluorescence detector reconstruction measured on an event-by-event basis, and show that the data taken over the past seven years with AERA shows a light cosmic-ray mass composition reconstruction in the energy range from $10^{17.5}$ to $10^{18.8} \mathrm{eV}$.

\footnotetext{
*Presenter
} 


\section{Introduction}

The types of particles cosmic rays consist of is key to understanding the sources, acceleration mechanisms, and propagation. At energies above the knee, one has to rely on indirect detections of cosmic rays and mass composition-sensitive parameters and on models to reconstruct the mass composition. Of particular interest is the energy regime around $10^{17}$ to $10^{18} \mathrm{eV}$, where it is suspected that the origin of the cosmic-ray flux transitions from Galactic to extragalactic sources, leading to a change of mass composition. AERA measures the radio emission of extensive air showers in the energy of $10^{17}$ to $10^{19} \mathrm{eV}$, probing a different aspect of the shower physics with respect to fluorescence or Cherenkov-light measurements. It is thus a valuable tool to verify our understanding of air shower physics. In recent years there have been many improvements to the understanding of radio emission mechanisms and reconstruction of radio signals. Here, we discuss the improvements to the reconstruction of $X_{\max }$ at AERA, our understanding of the systematic uncertainties, and show the results of the mass composition study from seven years of AERA data, also including an event-to-event comparison of showers measured with both the fluorescence detector (FD) and AERA.

\section{Radio Detection with AERA}

The Auger Engineering Radio Array (AERA) was developed as an enhancement of the Pierre Auger Observatory to measure radio emission of cosmic rays in the energy range of $10^{17}-10^{19} \mathrm{eV}$ [1]. With 153 antenna stations, spread over an area of about $17 \mathrm{~km}^{2}$, AERA is currently the largest radio array in the world for the study of cosmic rays. In the electromagnetic part of an air shower, radiation is produced from the movement of electric charges. This results in a thin radiation front propagating towards the ground that is detected as a short sharp pulse of a few nanoseconds at $\mathrm{MHz}$ to $\mathrm{GHz}$ frequencies that is strongest in the $\mathrm{MHz}$ regime. It has been shown that there is relation between the emission of the radio signal and the energy of the primary cosmic ray [2], such that the measured footprint can be used as a calorimetric estimation of the cosmic-ray energy, but also that the information about the primary cosmic-ray particle type is captured in the footprint. Heavier particles will interact earlier in the atmosphere and thus, for geometrical reasons, will give a more extended footprint on the ground than light particles. The width of the footprint can thus be considered a probe for the particle type [13].

\section{Selection of Air Showers}

To achieve a high-quality dataset of air shower reconstructions, first a new data-quality monitoring tool was created that can automatically identify periods of radio antenna malfunction or excessive RFI noise. This tool treats each type of hardware configuration separately to define a nominal operating regime based on a set of quantities derived from the voltage time traces and frequency spectra. If malfunctions in a station are persistent or reoccurring, then these are identified and logged as periods of bad operation and the station is automatically rejected during the reconstruction of air showers in the Offline framework [9]. This step makes sure that the reconstruction quality does not deteriorate, which could lead to failed or skewed reconstructed air shower 
properties. Then, a set of quality cuts was defined to reject low-quality shower reconstructions. The resulting dataset consists of 2153 air showers measured with the surface detector (SD) and AERA between roughly $10^{17}$ and $10^{19} \mathrm{eV}$, with zenith angles below $55^{\circ}$, and with at least 5 radio stations with signal to noise ratio $\geq 10$. A subset of 53 showers, that also have been reconstructed with FD, have been selected by the criterion that it should have a good FD $X_{\max }$ reconstruction on an event-by-event level, such that these can be used as an independent check on the AERA $X_{\max }$ reconstruction.

\section{4. $X_{\max }$ Reconstruction Method}

The reconstruction of the particle type (atomic mass, or simply mass) of ultra high energy cosmic rays relies on mass-sensitive parameters of the created extensive air showers since the primary particle, in its initial state, is lost in the first interaction. Measuring this with radio antennas can be done because the radio emission is extended along the entire shower and thus contains the information of the entire shower, that in turn is determined by the initial particle. This masssensitivity has been investigated in the past, using several methods that relate some property of the radio signal to the depth of the shower maximum $\left(X_{\max }\right)$ [2]. The $X_{\max }$ values can be related back to the atomic masses of the particles using CORSIKA air shower simulations.

For our $X_{\max }$ reconstruction method, we build upon the method developed for LOFAR by Buitink (2014) [3]. We set up CORSIKA/CoREAS v7.7100 [10,11] simulations using QGSJetII04 [12] to match, as accurately as possible, the conditions of the measured air showers (thinning of $10^{-6}$ is used), accounting for the time-dependent GDAS atmospheric models using the gdastool [4], a time-dependent geomagnetic field model, and an inclined AERA detector plane with additional virtual radio antennas such that the radio footprint signal can be interpolated at any point.

For each of the 2153 high-quality AERA air showers we created 27 dedicated air shower simulations, 12 induced by iron nuclei and 15 by protons. Our method uses the reconstructed arrival direction, core position, and primary energy of high-quality AERA showers as input to simulate this set of air showers simulations that spans the parameter space of the mass of the initial particle. The radio signals in these simulated showers are then reconstructed within the Offline framework to resemble air showers that would be measured by AERA, i.e. including detector effects.

By comparing the energy fluences $u$, the integrated signal of the cosmic-ray pulses, reconstructed from AERA, to energy fluences reconstructed from air showers simulations, a likelihood measure has been defined to quantify their similarity:

$$
\chi^{2}=\sum_{\text {stations }} \frac{\left(u_{\text {measured }}-S \cdot u_{\text {simulated }}\left(\vec{r}_{\text {shift }}\right)\right)^{2}}{\sigma_{u_{\text {measured }}}^{2}},
$$

where there are 3 free parameters: a scaling factor $S$ for the energy fluence is introduced that can account for the uncertainty in the SD energy estimate and any systematic uncertainties on the energy scale, the absolute predictions of the simulation in CORSIKA, and the reconstruction of the simulated radio signals. Also introduced is a core shift to account for the uncertainty in the AERA core estimate.

A minimizing procedure has been developed to estimate $X_{\max }$ from the minimum of a parabola fit to the $\chi^{2} / \operatorname{ndf}\left(X_{\max }\right)$ values of the simulations, while accounting for statistical and systematic 

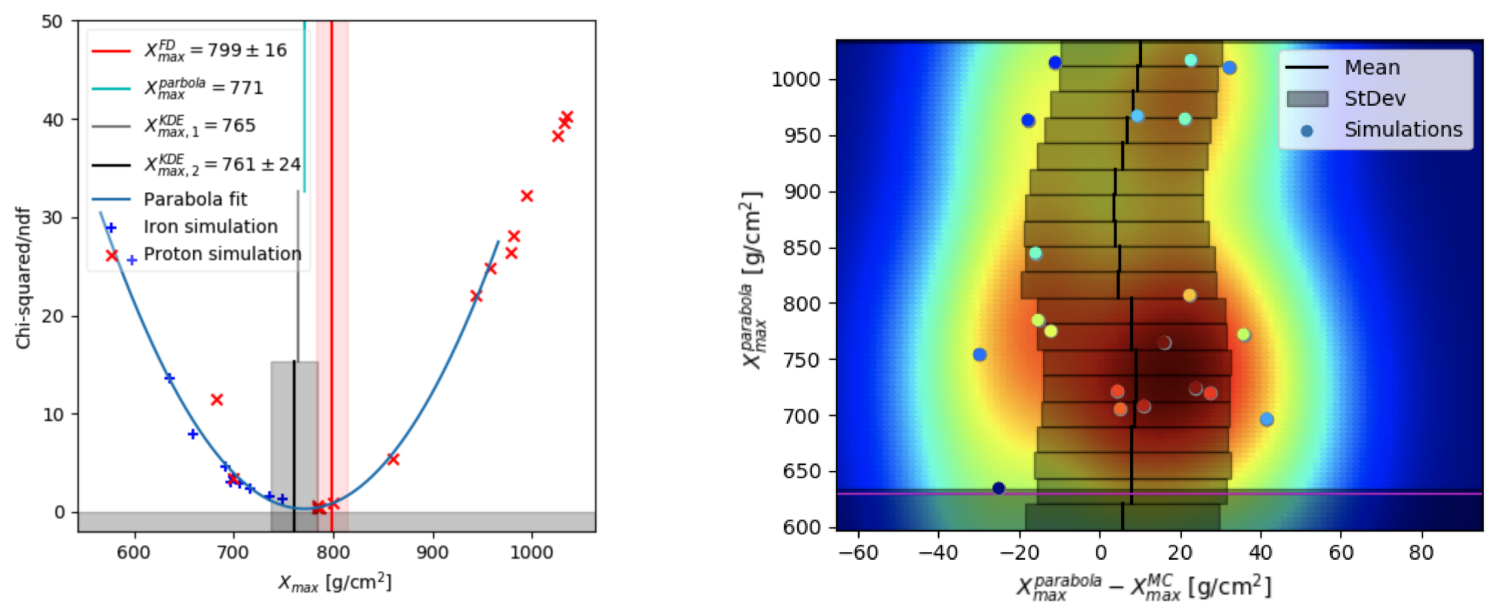

Figure 1: (Left): A parabola fit to the reduced chi-squared values between each of the simulated showers and the measured shower (red and blue points). The $X_{\max }$ value at the minimum of the parabola (cyan line) is corrected for bias resulting in the intermediate-step $X_{\max , 1}^{\mathrm{KDE}}$ estimate (gray line) and the final $X_{\max , 2}^{\mathrm{KDE}} \equiv X_{\max }^{\mathrm{AERA}}$ estimator (black line, with uncertainty $\sigma X_{\max }^{\mathrm{AERA}}$ ). For comparison, the independent FD-reconstructed $X_{\max }$ is plotted (red line, with uncertainty). (Right): Modelling of the uncertainty and bias in $X_{\max }$, as determined from reconstructing $X_{\max }$ for simulated showers for a particular shower measured with AERA.

uncertainties in the form of the three free parameters. The scipy basinhoppin algorithm is used to iteratively minimize for chi-squared, at each step fitting the $X_{\max }$-parabola, until a global minimum is found. An example of the fit for the found global minimum for one air shower is shown in Figure 1 (left).

The $X_{\max }$ values of the simulated showers themselves are also reconstructed to evaluate the quality of the reconstruction. This provides an estimation on the uncertainty of each reconstructed $X_{\max }$, but also allows for the identification and correction of systematic effects in the parabola fit estimator of $X_{\max }$, which was not accounted for in previous works. This might be of some importance for other experiments, performing similar analyses, especially for those similarly limited in the amount of antennas per measured air shower. This method can also account for limitations specific to sparse radio arrays with multiple irregularly-spaced antenna grids and with multiple antenna types (such as AERA). If not treated properly, this can bias the $X_{\max }$ reconstruction and affect the estimation of the uncertainty on $X_{\max }$.

Figure 1 (right) shows the reconstruction of $X_{\max }$ as function of the difference to the true MonteCarlo value for the simulations of one particular AERA shower. The spread of the points indicates the resolution in $X_{\max }$. Any significant deviation from $\Delta X_{\max }=0$ indicates that the $X_{\max }^{\text {parabola }}$ estimation was not bias-free. A two-dimensional probability distribution is constructed from these points, using a kernel density estimator (KDE) which is plotted in the figure as the colored background. At each parabola $X_{\max }$ value a slice of the KDE can be taken as one-dimensional probability density function (PDF) for the spread and deviation from the true $X_{\max }$. At regular spacing in parabola $X_{\max }$, the mean and the uncertainty values are plotted as gray bands. This KDE procedure is first performed while keeping the free parameters at the Monte-Carlo-true values to only identify the bias caused by the parabola fitting. This is corrected for w.r.t. to the parabola- $X_{\max }$ estimate to 


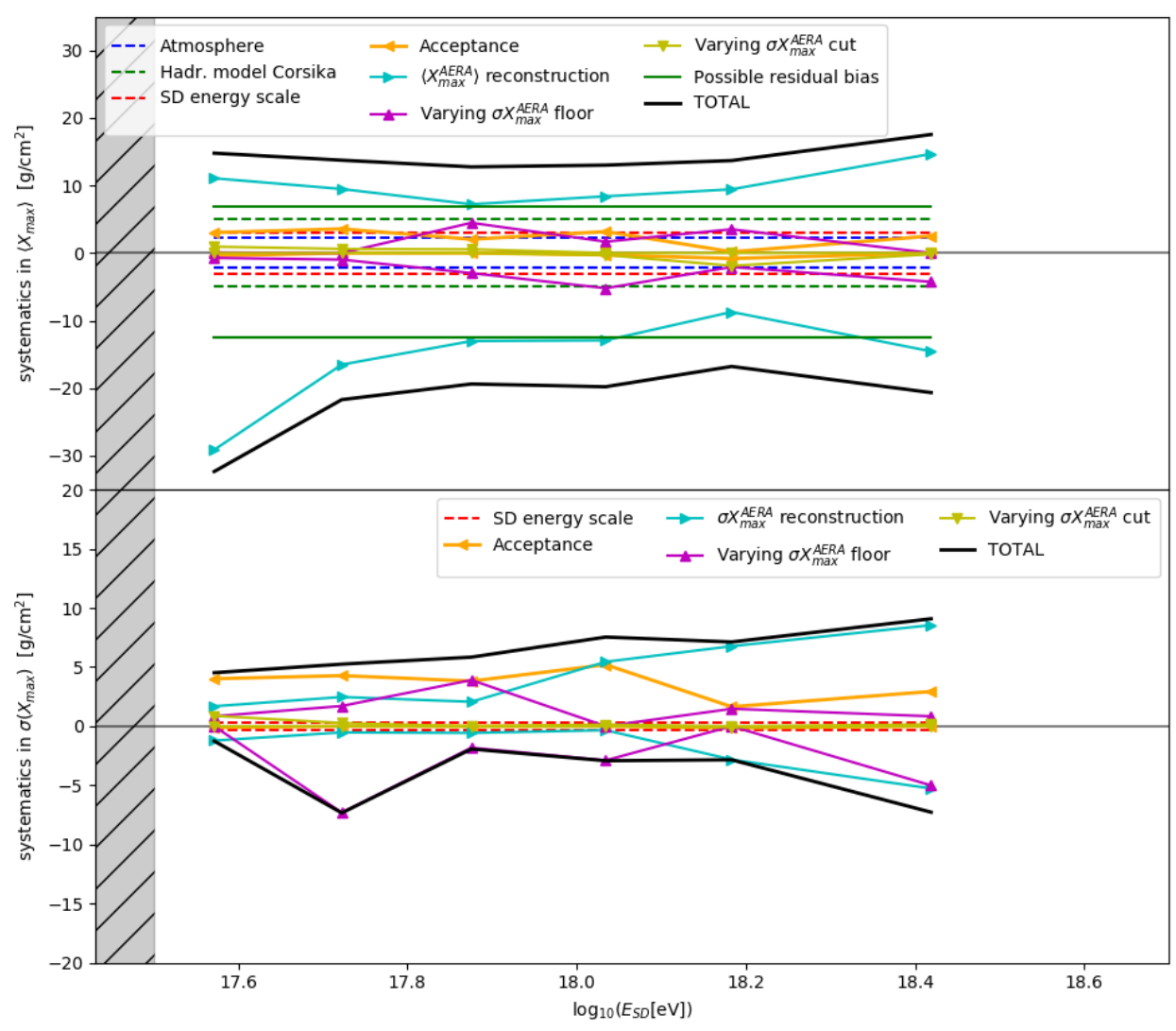

Figure 2: (Top): Overview of systematic uncertainties on the mean of the $X_{\max }$ distribution $\left(\left\langle X_{\max }\right\rangle\right)$. (Bottom): Overview of systematic uncertainties on the natural spread of $X_{\max }\left(\sigma\left(X_{\max }\right)\right)$. Dashed lines are constant contributions inherent to the simulation of the air showers. The solid lines are systematic uncertainties calculated for the AERA dataset.

construct an improved $X_{\max }$ estimator $X_{\max , 1}^{\mathrm{KDE}}$ (see Figure 1 (left)). Next, this is repeated without fixing the free parameters to account and correct for any remaining bias caused by this freedom, providing then the final $X_{\max }$ estimator $X_{\max , 2}^{\mathrm{KDE}} \equiv X_{\max }^{\mathrm{AERA}}$ and the total uncertainty on this value (see Figure 1 (left)).

\section{Systematic Uncertainties on $X_{\max }$}

Figure 2 shows an overview of systematic uncertainties on the mean and width of the $X_{\max }$ distribution as function of energy. It includes uncertainties from the choice of hadronic interaction model [3], implementation of the GDAS atmosphere in CORSIKA [3, 4] and the energy scale from SD [5]. Next also the effects of the event selection are accounted for. The $X_{\max }$ reconstruction of simulated air showers is used to cut on the acceptance, requiring that at least $90 \%$ of an iron or a proton Gumbel $X_{\max }$ distribution at the energy of the particular shower would have been detected by AERA. The acceptance to showers from iron nuclei is $100 \%$; the radio footprints of those showers are wider and easier to detect than for lighter particles. For protons the acceptance is $100 \%$ up to roughly 800 to $850 \mathrm{~g} / \mathrm{cm}^{2}$ for energies between $10^{17.5}$ to $10^{18.5} \mathrm{eV}$, respectively, and decreases 

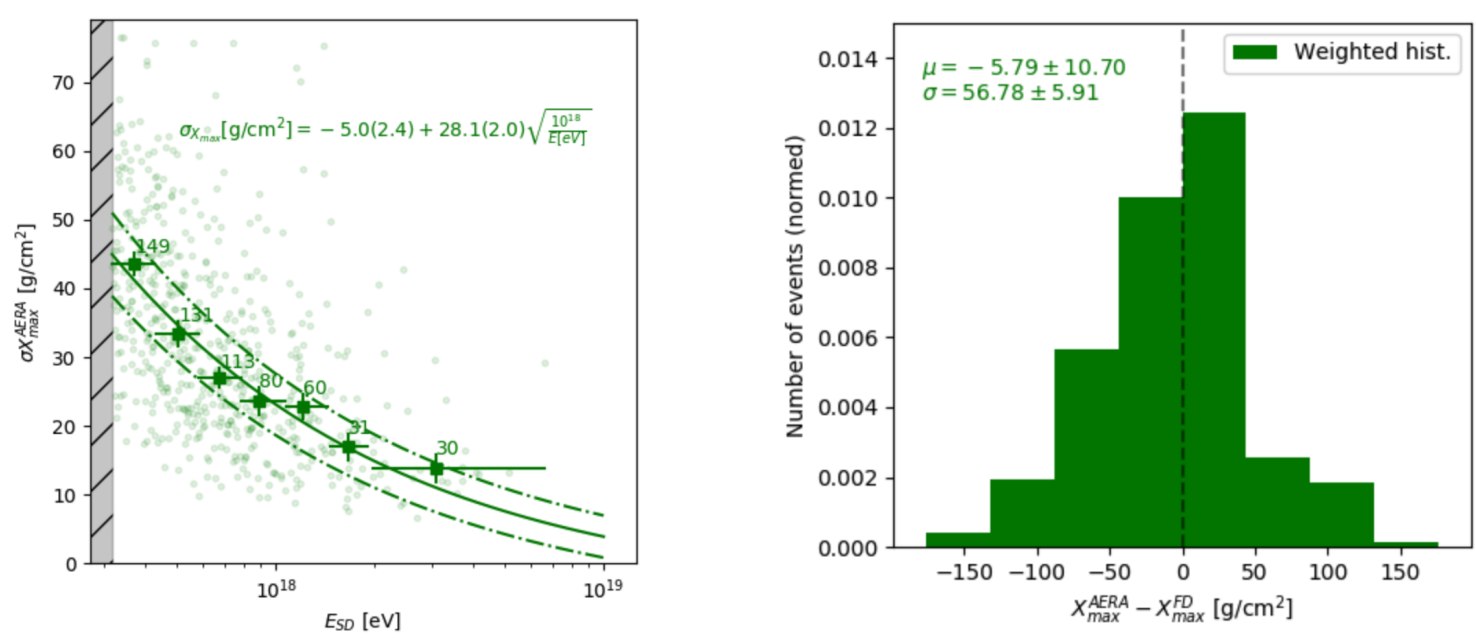

Figure 3: (Left): Resolution of the $X_{\max }^{\mathrm{AERA}}$ reconstruction method as a function of energy in units of column density. Plotted are the uncertainties on $X_{\max }$ for all showers after quality cuts (circles), the median values after binning (squares), and a generic function scaling with $E^{-0.5}$ fitted to parametrize the average resolution (solid line with $1 \sigma$-confidence bands). The fitted function is quoted in the figure. (Right): Results of the comparison of $X_{\max }$ for showers measured with both FD and AERA.

slowly at higher $X_{\max }$. Any residual bias on the mean and width of the $X_{\max }$ distribution from this is calculated to be less than $3 \mathrm{~g} / \mathrm{cm}^{2}$ and $4 \mathrm{~g} / \mathrm{cm}^{2}$, respectively when assuming the least favourable case of a pure proton composition. The possible bias is taken as systematic uncertainty. Next, the systematic bias from our $X_{\max }$ reconstruction method is evaluated using the CORSIKA simulations, calculating the effect of the method on the mean and width of a pure proton and pure iron Gumbel $X_{\max }$ distribution versus the simulation-reconstructed $X_{\max }$ distributions. Two additional minor contributions to the systematic uncertainty are shown that arise from the calculation of $\left\langle X_{\max }\right\rangle$ and $\sigma\left(X_{\max }\right)$. The first originates from setting a minimum uncertainty on each reconstructed $X_{\max }$ value. This is $15 \mathrm{~g} / \mathrm{cm}^{2}$ by default, then varied between $10 \mathrm{~g} / \mathrm{cm}^{2}$ and $20 \mathrm{~g} / \mathrm{cm}^{2}$, and the change in the results is taken as a contribution to the systematic uncertainty. The second minor contribution stems from setting a limit on the maximum uncertainty on any reconstructed $X_{\max }$. Such a cut can affect the natural spread in $X_{\max }$, so it is varied and the range of results are taken as systematic uncertainty. This uncertainty then accounts for the effects of low-number statistics. Finally, the presence of a possible residual bias is investigated by evaluating the mean $X_{\max }$ (taking into account the expected increase of $X_{\max }$ with energy) as function of geometry parameters such as the arrival direction and core position on which it should not depend if AERA were 100\% sensitive. An upper limit on residual bias has been determined from this and is shown in Figure 2.

\section{Resolution on $X_{\max }$}

Figure 3 (left) shows the uncertainties on $X_{\max }^{\mathrm{AERA}}$ for all reconstructed $X_{\max }^{\mathrm{AERA}}$ values (circles) for showers after quality and acceptance cuts. Plotted with green squares is the median resolution in $X_{\max }^{\mathrm{AERA}}$. A generic function scaling with $E^{-0.5}$ has been fitted (solid line with $1 \sigma$-confidence bands). The improvement of the resolution with energy is mainly driven by the increase of the radio 

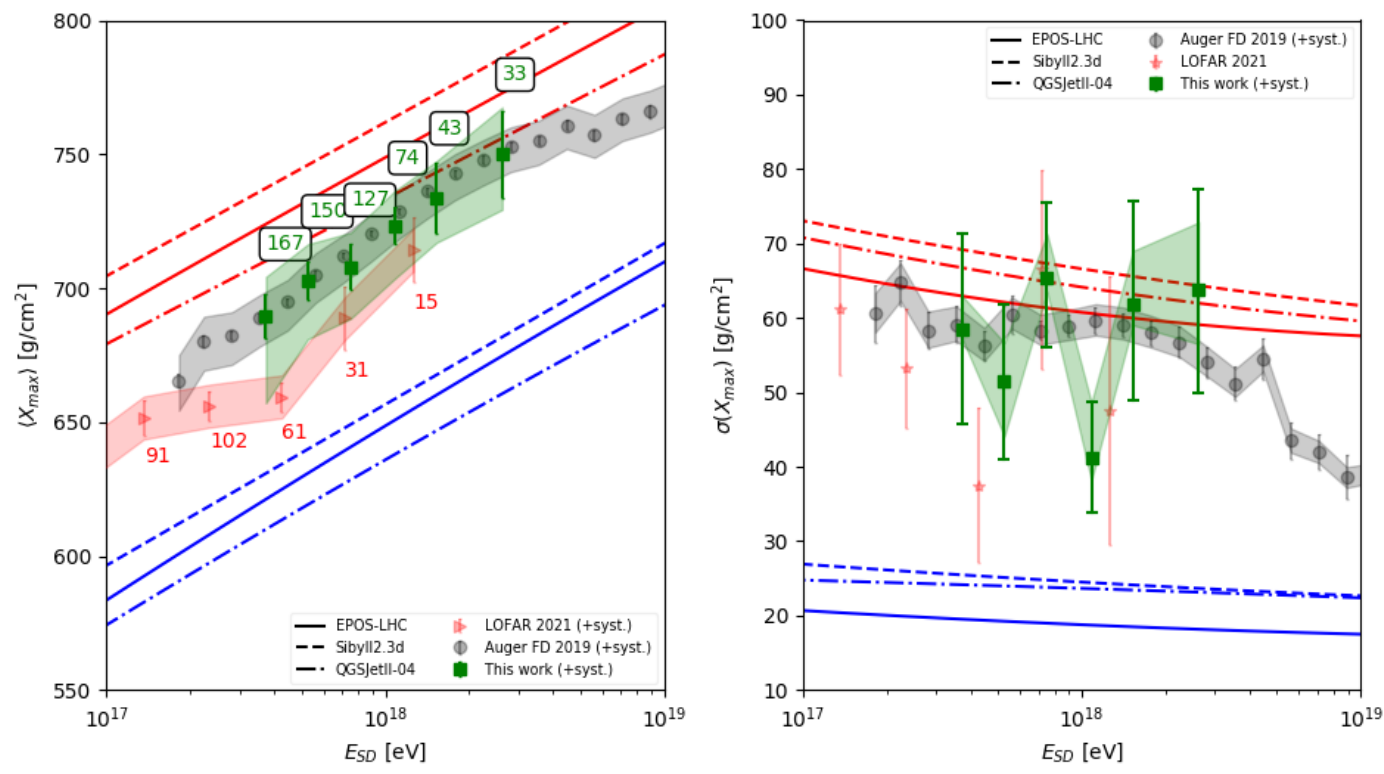

Figure 4: Mean (left) and standard deviation (right) of the $X_{\max }$ distribution as measured by AERA in this work (green). The results are compared to predictions from CORSIKA air shower simulations for multiple hadronic interaction models (lines) for proton (red) and iron (blue) mass compositions [6] and compared to measurements by LOFAR [8] and Auger FD [6]. The statistical uncertainties are plotted as error bars and the systematic uncertainties as bands if available.

signals at higher energies. For comparison, the resolution for FD is $25 \mathrm{~g} / \mathrm{cm}^{2}$ at $E=10^{17.8} \mathrm{eV}$ and improves to $15 \mathrm{~g} / \mathrm{cm}^{2}$ at higher energies [6]. The resolution of the radio- $X_{\max }$ method presented in this work is thus quite competitive. Compared to the radio template-fitting performed at LOFAR, similar resolutions are achieved if one compares the resolution at the energies where both detectors see showers with sufficient radio stations. LOFAR reported an average resolution of $19 \mathrm{~g} / \mathrm{cm}^{2}$ for showers with energies between $10^{16.8}$ and $10^{18.3} \mathrm{eV}$ [8]. AERA reaches the same average resolution at energies of $10^{18} \mathrm{eV}$, in spite of the much sparser antenna grid. If one compares the $X_{\max }$ resolution of this method to other methods or experiments it should be kept in mind that we find a clear relation between the resolution in $X_{\max }$ and the energy of the showers. A direct comparison of single quoted values for average resolutions, as has been the norm in the past for the radio experiments with typically small sets of air showers, should thus generally be avoided or at least this strong energy dependence should be kept in mind.

\section{Radio versus fluorescence $X_{\max }$}

Figure 3 (right) shows the results of the comparison of 53 air showers with independent $X_{\max }$ reconstructions by FD and AERA. It shows the distribution of the differences in $X_{\max }$ for the two methods on an event-to-event basis. The $X_{\max }$ for both have been independently reconstructed, so the FD values function as an independent validation of the $X_{\max }^{\mathrm{AERA}}$ reconstruction. It shows that there is no significant systematic bias between AERA and FD and sets an upper limit on the systematic uncertainty on $X_{\max }$, for showers with energies around $10^{17.5}-10^{18} \mathrm{eV}$, of $X_{\max }^{\mathrm{AERA}}-X_{\max }^{\mathrm{FD}}=$ 
$-5.29 \pm 10.79 \mathrm{~g} / \mathrm{cm}^{2}$. The combined mean resolution $\left(56.78 \pm 5.91 \mathrm{~g} / \mathrm{cm}^{2}\right)$ matches the individual mean uncertainties of FD and AERA for these showers $\left(18.5 \pm 1.4 \mathrm{~g} / \mathrm{cm}^{2}\right.$ and $50.8 \pm 6.0 \mathrm{~g} / \mathrm{cm}^{2}$, respectively).

\section{The $X_{\max }$ distribution from AERA}

In Figure 4 we calculate the mean and width of the AERA $X_{\max }$ distribution using a bias free sample of 594 showers above $E=10^{17.5} \mathrm{eV}$. The AERA $X_{\max }$ results are interpreted in terms of the types of the cosmic-ray particles, using three different hadronic interaction models. Our results provide new independent evidence for a light-mixed composition at $E=10^{17.5} \mathrm{eV}$, that becomes even lighter towards energies of $10^{18.5} \mathrm{eV}$. We show good agreement with the FD results, both in the mean and the width of the $X_{\max }$ distribution. Comparing our results to the LOFAR radio experiment, we note that the discrepancy between FD and LOFAR previously left open the possibility of a systematic shift in $X_{\max }$ due to the difference between radio and fluorescence methods. The AERA $X_{\max }$ composition now shows a lighter composition, in agreement with FD, suggesting the radio technique is not generally biased w.r.t. fluorescence. Our investigation of the systematic effects for AERA include the systematic contributions reported by LOFAR [8] and while the $X_{\max }$ results of the two experiments are compatible within the quoted uncertainty bands, some of the contributions are likely to be correlated such that further investigation is still required.

\section{Conclusions}

We have presented an improved reconstruction method for AERA and made an extensive investigation of the systematic uncertainties, highlighting the latter is key to understand and compare $X_{\max }$ measurements. We have shown the $X_{\max }$ resolution as a function of energy where a precision of up to $15 \mathrm{~g} / \mathrm{cm}^{2}$ is achieved. Comparison of a subset of showers measured simultaneously with AREA and FD shows no significant bias in the former AERA $X_{\max }$ reconstruction method, providing support for both $X_{\max }$ reconstructions. Analysis of the full seven years of AREA measurements show a light composition between $10^{17.5}$ and $10^{18.8} \mathrm{eV}$ fully compatible with FD measurements.

\section{References}

[1] P. Abreu [for the Pierre Auger Collaboration], J. Instrum. 7 (2012) P10011

[2] F. G. Schröder et al., Bull. Am. Astron. Soc. 51 (2019) 131

[3] S. Buitink et al., Nature 531 (2016) 70

[4] P. Mitra et al., Astropart. Phys. 123 (2020) 102470

[5] B. R. Dawson [for the Pierre Auger Collaboration], PoS(ICRC2019)274

[6] A. Yushkov [for the Pierre Auger Collaboration], PoS(ICRC2019)482

[7] S. Buitink et al., Phys. Rev. D 90 (2014) 082003

[8] A. Corstanje et al., Phys. Rev. D 103 (2021) 10, 102006

[9] P. Abreu [for the Pierre Auger Collaboration], Nucl. Instrum. Meth. A 635 (2011) 92-102

[10] D. Heck et al., FZKA Tech. Umw. Wis. B 6019 (1998)

[11] T. Huege et al., AIP Conf. Proc. 1535 (2013) 128-132

[12] S. Ostapchenko, Nucl. Phys. B-Proc. Sup. 151 (2006) 143-146

[13] E. Holt [for the Pierre Auger Collaboration], PoS(ICRC2019)280 


\section{The Pierre Auger Collaboration}

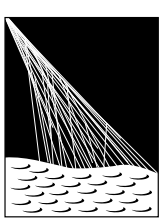

PIERRE

AUSGERVATORY

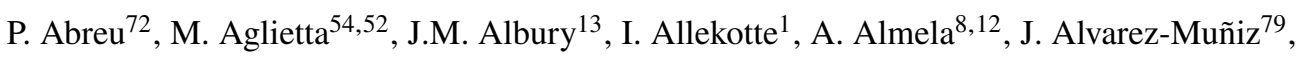
R. Alves Batista ${ }^{80}$, G.A. Anastasi ${ }^{63,52}$, L. Anchordoqui ${ }^{87}$, B. Andrada ${ }^{8}$, S. Andringa ${ }^{72}$, C. $\mathrm{Aramo}^{50}$, P.R. Araújo Ferreira ${ }^{42}$, J. C. Arteaga Velázquez ${ }^{67}$, H. Asorey $^{8}$, P. Assis ${ }^{72}$, G. Avila ${ }^{11}$, A.M. Badescu ${ }^{75}$, A. Bakalova ${ }^{32}$, A. Balaceanu ${ }^{73}$, F. Barbato ${ }^{45,46}$, R.J. Barreira Luz $^{72}$, K.H. Becker ${ }^{38}$, J.A. Bellido ${ }^{13,69}$, C. Berat ${ }^{36}$, M.E. Bertaina ${ }^{63,52}$, X. Bertou ${ }^{1}$, P.L. Biermann ${ }^{b}$, V. Binet ${ }^{6}$, K. Bismark ${ }^{39,8}$, T. Bister ${ }^{42}$, J. Biteau ${ }^{37}$, J. Blazek ${ }^{32}$, C. Bleve ${ }^{36}$, M. Boháčová ${ }^{32}$, D. Boncioli ${ }^{57,46}$, C. Bonifazi ${ }^{9,26}$, L. Bonneau Arbeletche ${ }^{21}$, N. Borodai ${ }^{70}$, A.M. Botti ${ }^{8}$, J. Brack ${ }^{d}$, T. Bretz ${ }^{42}$, P.G. Brichetto Orchera ${ }^{8}$, F.L. Briechle ${ }^{42}$, P. Buchholz ${ }^{44}$, A. Bueno ${ }^{78}$, S. Buitink ${ }^{15}$, M. Buscemi ${ }^{47}$, M. Büsken ${ }^{39,8}$, K.S. Caballero-Mora ${ }^{66}$, L. Caccianiga ${ }^{59,49}$, F. Canfora ${ }^{80,81}$, I. Caracas ${ }^{38}$, J.M. Carceller ${ }^{78}$, R. Caruso ${ }^{58,47}$, A. Castellina ${ }^{54,52}$, F. Catalani ${ }^{19}$, G. Cataldi ${ }^{48}$, L. Cazon ${ }^{72}$, M. Cerda ${ }^{10}$, J.A. Chinellato ${ }^{22}$, J. Chudoba ${ }^{32}$, L. Chytka ${ }^{33}$, R.W. Clay ${ }^{13}$, A.C. Cobos Ceruttii ${ }^{7}$, R. Colalillo ${ }^{60,50}$, A. Coleman ${ }^{93}$, M.R. Coluccia ${ }^{48}$, R. Conceição ${ }^{72}$, A. Condorelli ${ }^{45,46}$, G. Consolati ${ }^{49,55}$, F. Contreras ${ }^{11}$, F. Convenga ${ }^{56,48}$, D. Correia dos Santos $^{28}$, C.E. Covault ${ }^{85}$, S. Dasso ${ }^{5,3}$, K. Daumiller ${ }^{41}$, B.R. Dawson ${ }^{13}$, J.A. Day ${ }^{13}$, R.M. de Almeida $^{28}$, J. de Jesús ${ }^{8,41}$, S.J. de Jong ${ }^{80,81}$, G. De Mauro ${ }^{80,81}$, J.R.T. de Mello Neto ${ }^{26,27}$, I. De Mitri ${ }^{45,46}$, J. de Oliveira ${ }^{18}$, D. de Oliveira Franco ${ }^{22}$, F. de Palma ${ }^{56,48}$, V. de Souza $^{20}$, E. De Vito ${ }^{56,48}$, M. del Río ${ }^{11}$, O. Deligny ${ }^{34}$, L. Deval ${ }^{41,8}$, A. di Matteo $^{52}$, C. Dobrigkeit ${ }^{22}$, J.C. D’Olivo ${ }^{68}$, L.M. Domingues Mendes ${ }^{72}$, R.C. dos Anjos ${ }^{25}$, D. dos Santos $^{28}$, M.T. Dova ${ }^{4}$, J. Ebr ${ }^{32}$, R. Engel ${ }^{39,41}$, I. Epicoco ${ }^{56,48}$, M. Erdmann ${ }^{42}$, C.O. Escobar ${ }^{a}$, A. Etchegoyen ${ }^{8,12}$, H. Falcke ${ }^{80,82,81}$, J. Farmer ${ }^{92}$, G. Farrar ${ }^{90}$, A.C. Fauth ${ }^{22}$, N. Fazzini ${ }^{a}$, F. Feldbusch ${ }^{40}$, F. Fenu ${ }^{54,52}$,

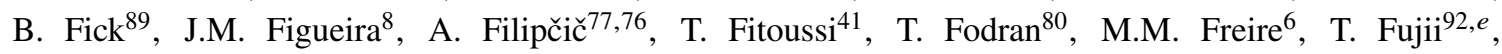
A. Fuster $^{8,12}$, C. Galea ${ }^{80}$, C. Galelli ${ }^{59,49}$, B. García ${ }^{7}$, A.L. Garcia Vegas ${ }^{42}$, H. Gemmeke ${ }^{40}$, F. Gesualdi ${ }^{8,41}$, A. Gherghel-Lascu ${ }^{73}$, P.L. Ghia ${ }^{34}$, U. Giaccari ${ }^{80}$, M. Giammarchi ${ }^{49}$, J. Glombitza ${ }^{42}$, F. Gobbi ${ }^{10}$, F. Gollan ${ }^{8}$, G. Golup ${ }^{1}$, M. Gómez Berisso ${ }^{1}$, P.F. Gómez Vitale ${ }^{11}$, J.P. Gongora ${ }^{11}$, J.M. González ${ }^{1}$, N. González ${ }^{14}$, I. Goos ${ }^{1,41}$, D. Góra ${ }^{70}$, A. Gorgi ${ }^{54,52}$, M. Gottowik ${ }^{38}$, T.D. Grubb ${ }^{13}$, F. Guarino ${ }^{60,50}$, G.P. Guedes ${ }^{23}$, E. Guido ${ }^{52,63}$, S. $\mathrm{Hahn}^{41,8}$, P. $\mathrm{Hamal}^{32}$, M.R. Hampel ${ }^{8}$, P. Hansen ${ }^{4}$, D. Harari ${ }^{1}$, V.M. Harvey ${ }^{13}$, A. Haungs ${ }^{41}$, T. Hebbeker ${ }^{42}$, D. Heck ${ }^{41}$, G.C. Hill ${ }^{13}$, C. Hojvat ${ }^{a}$, J.R. Hörandel ${ }^{80,81}$, P. Horvath ${ }^{33}$, M. Hrabovský ${ }^{33}$, T. Huege ${ }^{41,15}$, A. Insolia ${ }^{58,47}$, P.G. Isar $^{74}$, P. Janecek ${ }^{32}$, J.A. Johnsen ${ }^{86}$, J. Jurysek ${ }^{32}$, A. Kääpä ${ }^{38}$, K.H. Kampert ${ }^{38}$, N. Karastathis ${ }^{41}$, B. Keilhauer ${ }^{41}$, J. Kemp ${ }^{42}$, A. Khakurdikar ${ }^{80}$, V.V. Kizakke Covilakam $^{8,41}$, H.O. Klages ${ }^{41}$, M. Kleifges ${ }^{40}$, J. Kleinfeller ${ }^{10}$, M. Köpke ${ }^{39}$, N. Kunka ${ }^{40}$, B.L. Lago ${ }^{17}$, R.G. Lang ${ }^{20}$, N. Langner ${ }^{42}$, M.A. Leigui de Oliveira ${ }^{24}$, V. Lenok $^{41}$, A. Letessier-Selvon ${ }^{35}$, I. LhenryYvon $^{34}$, D. Lo Presti ${ }^{58,47}$, L. Lopes ${ }^{72}$, R. López ${ }^{64}$, L. Lu ${ }^{94}$, Q. Luce ${ }^{39}$, J.P. Lundquist ${ }^{76}$, A. Machado Payeras $^{22}$, G. Mancarella ${ }^{56,48}$, D. Mandat ${ }^{32}$, B.C. Manning ${ }^{13}$, J. Manshanden ${ }^{43}$, P. Mantsch ${ }^{a}$, S. Marafico ${ }^{34}$, A.G. Mariazzi ${ }^{4}$, I.C. Mariş ${ }^{14}$, G. Marsella ${ }^{61,47}$, D. Martello ${ }^{56,48}$, S. Martinelli ${ }^{41,8}$, O. Martínez Bravo ${ }^{64}$, M. Mastrodicasa ${ }^{57,46}$, H.J. Mathes ${ }^{41}$, J. Matthews ${ }^{88}$, G. Matthiae ${ }^{62,51}$, E. Mayotte ${ }^{38}$, P.O. Mazur ${ }^{a}$, G. MedinaTanco $^{68}$, D. Melo $^{8}$, A. Menshikov ${ }^{40}$, K.-D. Merenda ${ }^{86}$, S. Michal ${ }^{33}$, M.I. Micheletti ${ }^{6}$, L. Miramonti ${ }^{59,49}$, S. Mollerach ${ }^{1}$, F. Montanet ${ }^{36}$, C. Morello ${ }^{54,52}$, M. Mostafá ${ }^{91}$, A.L. Müller ${ }^{8}$, M.A. Muller ${ }^{22}$, K. Mulrey ${ }^{15}$, R. Mussa ${ }^{52}$, M. Muzio ${ }^{90}$, W.M. Namasaka ${ }^{38}$, A. Nasr-Esfahani ${ }^{38}$, L. Nellen ${ }^{68}$, M. Niculescu-Oglinzanu ${ }^{73}$, M. Niechciol ${ }^{44}$, D. Nitz ${ }^{89}$, D. Nosek ${ }^{31}$, V. Novotny ${ }^{31}$, L. Nožka ${ }^{33}$, A Nucita ${ }^{56,48}$, L.A. Núñez ${ }^{30}$, M. Palatka ${ }^{32}$, J. Pallotta ${ }^{2}$, P. Papenbreer ${ }^{38}$, G. Parente ${ }^{79}$, A. Parra ${ }^{64}$, J. Pawlowsky ${ }^{38}$, M. Pech ${ }^{32}$, F. Pedreira ${ }^{79}$, J. Pȩkala ${ }^{70}$, R. Pelayo ${ }^{65}$, J. Peña-Rodriguez ${ }^{30}$, E.E. Pereira Martins ${ }^{39,8}$, J. Perez Armand ${ }^{21}$, C. Pérez

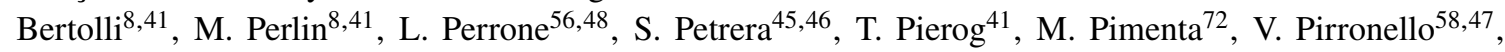
M. Platino ${ }^{8}$, B. Pont $^{80}$, M. Pothast ${ }^{81,80}$, P. Privitera ${ }^{92}$, M. Prouza ${ }^{32}$, A. Puyleart ${ }^{89}$, S. Querchfeld ${ }^{38}$, J. Rautenberg ${ }^{38}$, D. Ravignani ${ }^{8}$, M. Reininghaus ${ }^{41,8}$, J. Ridky ${ }^{32}$, F. Riehn ${ }^{72}$, M. Risse ${ }^{44}$, V. Rizi ${ }^{57,46}$, W. Rodrigues de Carvalho ${ }^{21}$, J. Rodriguez Rojo ${ }^{11}$, M.J. Roncoroni ${ }^{8}$, S. Rossoni ${ }^{43}$, M. Roth ${ }^{41}$, E. Roulet ${ }^{1}$, A.C. Rovero ${ }^{5}$, P. Ruehl ${ }^{44}$, A. Saftoiu ${ }^{73}$, F. Salamida ${ }^{57,46}$, H. Salazar ${ }^{64}$, G. Salina ${ }^{51}$, J.D. Sanabria Gomez ${ }^{30}$, F. Sánchez ${ }^{8}$, E.M. Santos $^{21}$, E. Santos ${ }^{32}$, F. Sarazin ${ }^{86}$, R. Sarmento ${ }^{72}$, C. Sarmiento-Cano ${ }^{8}$, R. Sato ${ }^{11}$, 
P. Savina ${ }^{56,48,34,94}$, C.M. Schäfer ${ }^{41}$, V. Scherini ${ }^{56,48}$, H. Schieler ${ }^{41}$, M. Schimassek ${ }^{39,8}$, M. Schimp ${ }^{38}$, F. Schlüter ${ }^{41,8}$, D. Schmidt ${ }^{39}$, O. Scholten ${ }^{84,15}$, P. Schovánek ${ }^{32}$, F.G. Schröder ${ }^{93,41}$, S. Schröder ${ }^{38}$, J. Schulte ${ }^{42}$, S.J. Sciutto ${ }^{4}$, M. Scornavacche ${ }^{8,41}$, A. Segreto ${ }^{53,47}$, S. Sehgal ${ }^{38}$, R.C. Shellard ${ }^{16}$, G. Sigl ${ }^{43}$, G. Silli ${ }^{8,41}$, O. Sima ${ }^{73, f}$, R. Šmída ${ }^{92}$, P. Sommers ${ }^{91}$, J.F. Soriano ${ }^{87}$, J. Souchard ${ }^{36}$, R. Squartini ${ }^{10}$, M. Stadelmaier ${ }^{41,8}$, D. Stanca ${ }^{73}$, S. Staničc ${ }^{76}$, J. Stasielak ${ }^{70}$, P. Stassi ${ }^{36}$, A. Streich ${ }^{39,8}$, M. Suárez-Durán ${ }^{14}$, T. Sudholz ${ }^{13}$, T. Suomijärvi ${ }^{37}$, A.D. Supanitsky ${ }^{8}$, Z. Szadkowski ${ }^{71}$, A. Tapia ${ }^{29}$, C. Taricco ${ }^{63,52}$, C. Timmermans ${ }^{81,80}$, O. Tkachenko ${ }^{41}$, P. Tobiska ${ }^{32}$, C.J. Todero Peixoto ${ }^{19}$, B. Tomé ${ }^{72}$, Z. Torrès ${ }^{36}$, A. Travaini ${ }^{10}$, P. Travnicek ${ }^{32}$, C. Trimarelli ${ }^{57,46}$, M. Tueros ${ }^{4}$, R. Ulrich ${ }^{41}$, M. Unger ${ }^{41}$, L. Vaclavek ${ }^{33}$, M. Vacula ${ }^{33}$, J.F. Valdés Galicia ${ }^{68}$, L. Valore ${ }^{60,50}$, E. Varela ${ }^{64}$, A. Vásquez-Ramírez ${ }^{30}$, D. Veberič ${ }^{41}$, C. Ventura ${ }^{27}$, I.D. Vergara Quispe ${ }^{4}$, V. Verzi ${ }^{51}$, J. Vicha ${ }^{32}$, J. Vink ${ }^{83}$, S. Vorobiov ${ }^{76}$, H. Wahlberg ${ }^{4}$, C. Watanabe ${ }^{26}$, A.A. Watson ${ }^{c}$, M. Weber ${ }^{40}$, A. Weindl ${ }^{41}$, L. Wiencke ${ }^{86}$, H. Wilczyński ${ }^{70}$, M. Wirtz ${ }^{42}$, D. Wittkowski ${ }^{38}$, B. Wundheiler ${ }^{8}$, A. Yushkov ${ }^{32}$, O. Zapparrata ${ }^{14}$, E. Zas ${ }^{79}$, D. Zavrtanik ${ }^{76,77}$, M. Zavrtanik ${ }^{77,76}$, L. Zehrer ${ }^{76}$

${ }^{1}$ Centro Atómico Bariloche and Instituto Balseiro (CNEA-UNCuyo-CONICET), San Carlos de Bariloche, Argentina

${ }^{2}$ Centro de Investigaciones en Láseres y Aplicaciones, CITEDEF and CONICET, Villa Martelli, Argentina

${ }^{3}$ Departamento de Física and Departamento de Ciencias de la Atmósfera y los Océanos, FCEyN, Universidad de Buenos Aires and CONICET, Buenos Aires, Argentina

${ }^{4}$ IFLP, Universidad Nacional de La Plata and CONICET, La Plata, Argentina

${ }^{5}$ Instituto de Astronomía y Física del Espacio (IAFE, CONICET-UBA), Buenos Aires, Argentina

${ }^{6}$ Instituto de Física de Rosario (IFIR) - CONICET/U.N.R. and Facultad de Ciencias Bioquímicas y Farmacéuticas U.N.R., Rosario, Argentina

${ }^{7}$ Instituto de Tecnologías en Detección y Astropartículas (CNEA, CONICET, UNSAM), and Universidad Tecnológica Nacional - Facultad Regional Mendoza (CONICET/CNEA), Mendoza, Argentina

${ }^{8}$ Instituto de Tecnologías en Detección y Astropartículas (CNEA, CONICET, UNSAM), Buenos Aires, Argentina

${ }^{9}$ International Center of Advanced Studies and Instituto de Ciencias Físicas, ECyT-UNSAM and CONICET, Campus Miguelete - San Martín, Buenos Aires, Argentina

${ }^{10}$ Observatorio Pierre Auger, Malargüe, Argentina

${ }^{11}$ Observatorio Pierre Auger and Comisión Nacional de Energía Atómica, Malargüe, Argentina

12 Universidad Tecnológica Nacional - Facultad Regional Buenos Aires, Buenos Aires, Argentina

${ }^{13}$ University of Adelaide, Adelaide, S.A., Australia

14 Université Libre de Bruxelles (ULB), Brussels, Belgium

15 Vrije Universiteit Brussels, Brussels, Belgium

${ }^{16}$ Centro Brasileiro de Pesquisas Fisicas, Rio de Janeiro, RJ, Brazil

${ }^{17}$ Centro Federal de Educação Tecnológica Celso Suckow da Fonseca, Nova Friburgo, Brazil

${ }^{18}$ Instituto Federal de Educação, Ciência e Tecnologia do Rio de Janeiro (IFRJ), Brazil

${ }^{19}$ Universidade de São Paulo, Escola de Engenharia de Lorena, Lorena, SP, Brazil

${ }^{20}$ Universidade de São Paulo, Instituto de Física de São Carlos, São Carlos, SP, Brazil

${ }^{21}$ Universidade de São Paulo, Instituto de Física, São Paulo, SP, Brazil

22 Universidade Estadual de Campinas, IFGW, Campinas, SP, Brazil

${ }^{23}$ Universidade Estadual de Feira de Santana, Feira de Santana, Brazil

${ }^{24}$ Universidade Federal do ABC, Santo André, SP, Brazil

${ }^{25}$ Universidade Federal do Paraná, Setor Palotina, Palotina, Brazil

${ }^{26}$ Universidade Federal do Rio de Janeiro, Instituto de Física, Rio de Janeiro, RJ, Brazil

${ }^{27}$ Universidade Federal do Rio de Janeiro (UFRJ), Observatório do Valongo, Rio de Janeiro, RJ, Brazil

${ }^{28}$ Universidade Federal Fluminense, EEIMVR, Volta Redonda, RJ, Brazil

${ }^{29}$ Universidad de Medellín, Medellín, Colombia

${ }^{30}$ Universidad Industrial de Santander, Bucaramanga, Colombia

${ }^{31}$ Charles University, Faculty of Mathematics and Physics, Institute of Particle and Nuclear Physics, Prague, Czech Republic

32 Institute of Physics of the Czech Academy of Sciences, Prague, Czech Republic 
${ }^{33}$ Palacky University, RCPTM, Olomouc, Czech Republic

${ }^{34}$ CNRS/IN2P3, IJCLab, Université Paris-Saclay, Orsay, France

${ }^{35}$ Laboratoire de Physique Nucléaire et de Hautes Energies (LPNHE), Sorbonne Université, Université de Paris, CNRSIN2P3, Paris, France

${ }^{36}$ Univ. Grenoble Alpes, CNRS, Grenoble Institute of Engineering Univ. Grenoble Alpes, LPSC-IN2P3, 38000 Grenoble, France

${ }^{37}$ Université Paris-Saclay, CNRS/IN2P3, IJCLab, Orsay, France

38 Bergische Universität Wuppertal, Department of Physics, Wuppertal, Germany

${ }^{39}$ Karlsruhe Institute of Technology (KIT), Institute for Experimental Particle Physics, Karlsruhe, Germany

${ }^{40}$ Karlsruhe Institute of Technology (KIT), Institut für Prozessdatenverarbeitung und Elektronik, Karlsruhe, Germany

${ }^{41}$ Karlsruhe Institute of Technology (KIT), Institute for Astroparticle Physics, Karlsruhe, Germany

${ }^{42}$ RWTH Aachen University, III. Physikalisches Institut A, Aachen, Germany

${ }^{43}$ Universität Hamburg, II. Institut für Theoretische Physik, Hamburg, Germany

44 Universität Siegen, Department Physik - Experimentelle Teilchenphysik, Siegen, Germany

${ }^{45}$ Gran Sasso Science Institute, L'Aquila, Italy

46 INFN Laboratori Nazionali del Gran Sasso, Assergi (L'Aquila), Italy

${ }^{47}$ INFN, Sezione di Catania, Catania, Italy

${ }^{48}$ INFN, Sezione di Lecce, Lecce, Italy

${ }^{49}$ INFN, Sezione di Milano, Milano, Italy

${ }^{50}$ INFN, Sezione di Napoli, Napoli, Italy

51 INFN, Sezione di Roma "Tor Vergata", Roma, Italy

52 INFN, Sezione di Torino, Torino, Italy

53 Istituto di Astrofisica Spaziale e Fisica Cosmica di Palermo (INAF), Palermo, Italy

54 Osservatorio Astrofisico di Torino (INAF), Torino, Italy

55 Politecnico di Milano, Dipartimento di Scienze e Tecnologie Aerospaziali , Milano, Italy

56 Università del Salento, Dipartimento di Matematica e Fisica "E. De Giorgi”, Lecce, Italy

57 Università dell'Aquila, Dipartimento di Scienze Fisiche e Chimiche, L'Aquila, Italy

58 Università di Catania, Dipartimento di Fisica e Astronomia, Catania, Italy

${ }^{59}$ Università di Milano, Dipartimento di Fisica, Milano, Italy

${ }^{60}$ Università di Napoli "Federico II", Dipartimento di Fisica "Ettore Pancini”, Napoli, Italy

${ }^{61}$ Università di Palermo, Dipartimento di Fisica e Chimica "E. Segrè", Palermo, Italy

62 Università di Roma "Tor Vergata”, Dipartimento di Fisica, Roma, Italy

${ }^{63}$ Università Torino, Dipartimento di Fisica, Torino, Italy

64 Benemérita Universidad Autónoma de Puebla, Puebla, México

${ }^{65}$ Unidad Profesional Interdisciplinaria en Ingeniería y Tecnologías Avanzadas del Instituto Politécnico Nacional (UPIITA-IPN), México, D.F., México

66 Universidad Autónoma de Chiapas, Tuxtla Gutiérrez, Chiapas, México

${ }^{67}$ Universidad Michoacana de San Nicolás de Hidalgo, Morelia, Michoacán, México

${ }^{68}$ Universidad Nacional Autónoma de México, México, D.F., México

${ }^{69}$ Universidad Nacional de San Agustin de Arequipa, Facultad de Ciencias Naturales y Formales, Arequipa, Peru

${ }^{70}$ Institute of Nuclear Physics PAN, Krakow, Poland

${ }^{71}$ University of Łódź, Faculty of High-Energy Astrophysics,Lódź, Poland

${ }^{72}$ Laboratório de Instrumentação e Física Experimental de Partículas - LIP and Instituto Superior Técnico - IST, Universidade de Lisboa - UL, Lisboa, Portugal

73 "Horia Hulubei” National Institute for Physics and Nuclear Engineering, Bucharest-Magurele, Romania

${ }^{74}$ Institute of Space Science, Bucharest-Magurele, Romania

75 University Politehnica of Bucharest, Bucharest, Romania

76 Center for Astrophysics and Cosmology (CAC), University of Nova Gorica, Nova Gorica, Slovenia

${ }^{77}$ Experimental Particle Physics Department, J. Stefan Institute, Ljubljana, Slovenia

78 Universidad de Granada and C.A.F.P.E., Granada, Spain

${ }^{79}$ Instituto Galego de Física de Altas Enerxías (IGFAE), Universidade de Santiago de Compostela, Santiago de Compostela, Spain 
${ }^{80}$ IMAPP, Radboud University Nijmegen, Nijmegen, The Netherlands

${ }^{81}$ Nationaal Instituut voor Kernfysica en Hoge Energie Fysica (NIKHEF), Science Park, Amsterdam, The Netherlands

82 Stichting Astronomisch Onderzoek in Nederland (ASTRON), Dwingeloo, The Netherlands

${ }^{83}$ Universiteit van Amsterdam, Faculty of Science, Amsterdam, The Netherlands

${ }^{84}$ University of Groningen, Kapteyn Astronomical Institute, Groningen, The Netherlands

85 Case Western Reserve University, Cleveland, OH, USA

${ }^{86}$ Colorado School of Mines, Golden, CO, USA

${ }^{87}$ Department of Physics and Astronomy, Lehman College, City University of New York, Bronx, NY, USA

${ }^{88}$ Louisiana State University, Baton Rouge, LA, USA

${ }^{89}$ Michigan Technological University, Houghton, MI, USA

${ }^{90}$ New York University, New York, NY, USA

${ }^{91}$ Pennsylvania State University, University Park, PA, USA

92 University of Chicago, Enrico Fermi Institute, Chicago, IL, USA

93 University of Delaware, Department of Physics and Astronomy, Bartol Research Institute, Newark, DE, USA

94 University of Wisconsin-Madison, Department of Physics and WIPAC, Madison, WI, USA

${ }^{a}$ Fermi National Accelerator Laboratory, Fermilab, Batavia, IL, USA

${ }^{b}$ Max-Planck-Institut für Radioastronomie, Bonn, Germany

${ }^{c}$ School of Physics and Astronomy, University of Leeds, Leeds, United Kingdom

${ }^{d}$ Colorado State University, Fort Collins, CO, USA

${ }^{e}$ now at Hakubi Center for Advanced Research and Graduate School of Science, Kyoto University, Kyoto, Japan

$f$ also at University of Bucharest, Physics Department, Bucharest, Romania 